This is an author-created version for self-archiveing of the article:

A.R. Engert, F.Felten, H. Jelitto, G.A. Schneider

What do we know about surface charges on cracks in ferroelectric ceramics?

IUTAM Symposium on Multiscale Modelling of Fatigue, Damage and Fracture in Smart Materials, IUTAM Bookseries, 2011, Volume 24, pp. 9-20

DOI: 10.1007/978-90-481-9887-0_2

The final publication is available at www.springerlink.com:

https://springerlink3.metapress.com/content/k744045830590313/resource-

$\underline{\mathrm{se}-}$

cured/?target=fulltext.pdf\&sid=elkt5marabro3ga5t00bikmu\&sh=www.springerlin

k.com

\title{
What do we know about surface charges on cracks in ferroelectric ceramics?
}

\author{
Andrea R. Engert, Frank Felten, Hans Jelitto and Gerold A. Schneider
}

\begin{abstract}
The present work investigates the static and time dependent electric potential distribution around cracks in a poled ferroelectric ceramic by Kelvin Probe Force Microscopy (KFM). In a first step a Vickers indentation crack in poled lead zirconate titanate (PZT) was subjected to static electric fields of up to $500 \mathrm{~V} / \mathrm{mm}$ in poling direction, and the potential distribution around the crack was measured. In a second step, the polarity of the applied voltage was reversed against the poling direction during the measurement of the potential. Using a simple model, an effective dielectric constant of the crack, as well as the surface charge density on the crack face were calculated as a function of the distance from the crack tip, the applied field and the time. The results are discussed with reference to free charges on the crack surface, electrically induced domain switching at the crack tip and crack bridging.
\end{abstract}

A.R. Engert, H. Jelitto and G.A. Schneider Technische Universität Hamburg-Harburg, Institut für keramische Hochleistungswerkstoffe, Hamburg, Germany Phone: +49 40 42878-3037 Fax: -2647 Mail: g.schneider@tu-harburg.de www.tu-harburg.de/gk

F. Felten

Robert Bosch GmbH, Corporate Sector Research and Advance Engineering, Applied Research 1 - Materials, 70049 Stuttgart Germany 


\section{Introduction}

Ferroelectric materials are used, amongst other applications, in electro-mechanical transducer applications, converting mechanical forces into an electrical potential (direct piezoelectric effect) or vice versa (inverse piezoelectric effect). During their lifetime, ferroelectric ceramics must be capable to operate under long-term electro-mechanical loading. While they continue to find increasing use, their fracture mechanics are still investigated due to their low fracture toughness and complex material behavior. This work contributes to the understanding of the effect of electrical loading on fracture in ferroelectric ceramics.

A crack filled with air in a dielectric material with a high permittivity, is a void with a relative dielectric constant of one in a matrix with a dielectric constant that can be up to three orders of magnitude higher. If an electrical field is applied normal to the crack surface, it will penetrate the crack. The crack shape, the crack opening displacement and the ratio of the permittivities of the crack interior and the ceramic, will determine to what extent the electric flux through the crack will be diluted, and increased ahead of the tip. For cracks in a ferroelectric ceramic, i.e. in an electromechanically coupled material, this leads to a non-linear boundary value problem. In the literature three approaches are used to describe cracks in ferroelectric materials assuming impermeable boundaries (impermeable crack), permeable boundaries with an infinite dielectric constant of the crack (permeable crack), and permeable boundaries with a dielectric constant of the crack higher than one (semi-permeable crack). Theoretical works, e.g. from Balke et al [1] using the capacitor model of Hao and Shen [2] have shown that the latter results in the most realistic representation of the electric field around the crack. However, the capacitor model proved to be energetically not consistent [3]. In consequence, Landis $[4,5]$ derived the so called "energetically consistent" boundary conditions along the crack faces that include electrostatic tractions, which are caused by an electric field perpendicular to the crack faces closing the crack. His improved model, that also includes surface charges on the crack faces, and an electrical discharge model within the crack, predicts that an electrical load increases the critical mechanical load for fracture. Except of the permeable crack model, all other theoretical approaches predict that the electric field applied perpendicular to the crack plane increases the critical mechanical load for crack growth [6]. These theoretical predictions could not be observed experimentally. E.g. Jelitto et al. [7,8,9] and Häusler [10] show that DC electric fields of $1 / 3$ of the coercive field only influence the critical loads for crack growth to a very small extent.

Haug and McMeeking [11] showed, that screening charges on the crack surface would change the fracture behavior of the crack dramatically. Schneider et al. [12] already successfully used Kelvin Probe Force Microscopy (KFM) to study the potential distribution around an electrically loaded Vickers crack in PZT. From the results an effective dielectric constant was calculated for the crack tip. On the basis of a Griffith crack it was further shown, that a higher dielectric constant of the 
crack reduces the dependency of the crack tip energy release rate on an applied electric field. The present work is a continuation of the work presented by Schneider et al. [12]. It takes into account not only a single applied electric field, but different field strengths up to $500 \mathrm{~V} / \mathrm{mm}$. Besides, the potential distribution is not only analyzed at the crack tip, but to a crack length of around $60 \mu \mathrm{m}$. The objective was to analyze whether or not the crack could also be described by a single effective dielectric constant farther away from the tip and at different applied electric fields. This part of the work is described in more detail in the $\mathrm{PhD}$ thesis from Felten [13]. In addition, the time response of the electric potential distribution upon a reversal of the electric field against the polarization direction was studied. The motivation for this experiment was the assumption that free charges on the crack surface would not immediately change after the reversal of the applied voltage. The objective of all experiments was to verify the theoretical assumption of screening charges on the crack surface, trying to quantify and characterize them.

\section{Samples, experimental setup and methods}

KFM is a scanning probe technique, which allows mapping the surface potential of a sample versus the in-plane coordinates $\mathrm{x}$ and $\mathrm{y}$ in a two-pass technique. During the first pass the topography is recorded in tapping-mode. In the second pass, the so-called interleave scan, the topography is retraced in a set lift height and the contact potential difference is measured. As the tip travels over the sample surface in lift-mode, the tip and the cantilever experience a force wherever the potential on the surface is different from the potential of the tip. The force is nullified by varying the voltage of the tip so that the tip is at the same potential as the region of the sample surface underneath it. This voltage is plotted versus the in-plane $x-y-$ coordinates, creating the surface potential image. The principle of the KFM-mode is described e.g. by Kalinin and Bonnell [14], the resolution of KFM is discussed e.g. by Jacobs et al. [15].
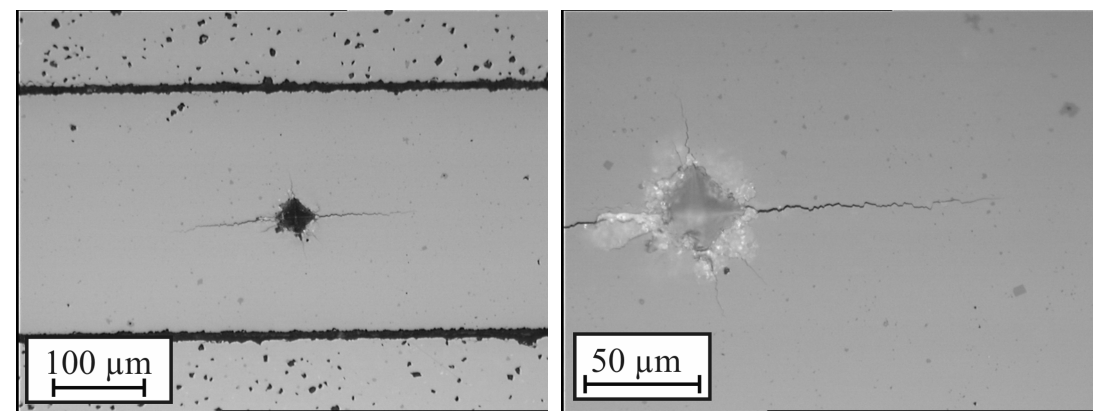

Fig. 1 Images of the Vickers indent taken with an optical microscope showing the two perpendicular crack paths. 
The experiments were performed in a similar way as described by Schneider et al. [12]. $270 \mu \mathrm{m}$ thin plates of a commercial soft PZT (Vibrit1100, Johnson Matthey) covered with thin gold electrodes on both sides were cut into samples of $1 \times 2 \mathrm{~cm}^{2}$. The plates were poled at room temperature with an electric field of $2 \mathrm{kV} / \mathrm{mm}$. The coercive field extracted from a hysteresis measurement (room temperature, $2 \mathrm{kV} / \mathrm{mm}$ maximum field, $8.3 \mathrm{mHz}$ ) is around $700 \mathrm{~V} / \mathrm{mm}$. According to the data sheet the dielectric constant $\varepsilon_{33}$ of the poled material is 4500 [16]. One thin $4 \mathrm{~cm} \times 260 \mu \mathrm{m}$ face was polished on a semi-automatic polishing machine (Saphir550, ATM GmbH) using diamond suspension down to a grain size of $0,25 \mu \mathrm{m}$ (DP-Suspension P on MD-NAP, Struers GmbH). The material's grain size measured with an optical microscope is around $3 \mu \mathrm{m}$. After polishing a Vickers indent $(0.5 \mathrm{~kg}, 10 \mathrm{~s}$, Vickers hardness tester 3212 preceding model of ZVH10, Zwick) was induced in the middle of the surface, producing cracks parallel to the surface edges (Fig. 1). The advantage of the indentation methodology was its easy control and simplicity concerning the experimental performance. Vickers indenters produce two basic types of crack systems: radial-median and lateral. After the diamond is removed, local residual stress states hold the cracks open [17, p.249ff.].

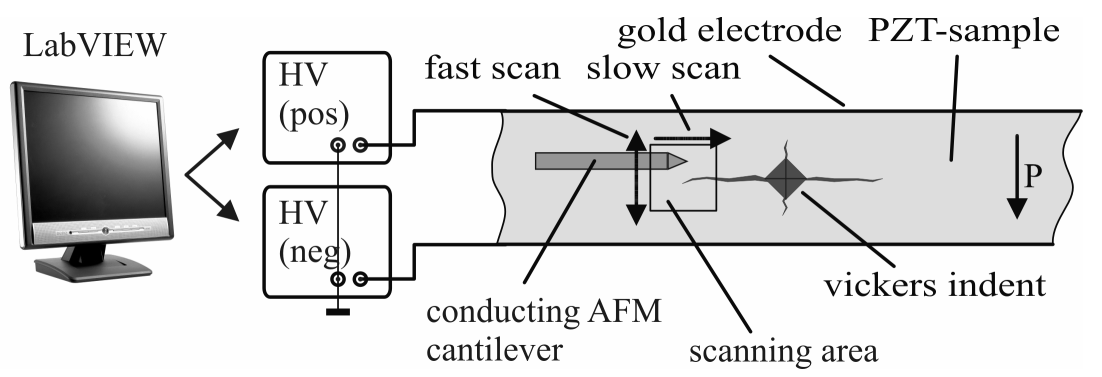

Fig. 2 Schematic of the experimental KFM setup.

The sample was fixed in a custom made holder and aligned under a Scanning Probe Microscope (SPM) in a way that the cantilever was perpendicular to the applied field, and would not electrostatically interact with the indent. The voltage was externally applied to the sample. For the measurements with constant fields two separate power supplies were used (HCN-35-12500, MCN-35-1250, FuG). For the measurements where the field was reversed, a bipolar power supply with two ports and a fast response (PZD700, large signal bandwidth up to $15 \mathrm{kHz}$, TREK INC.) was chosen. The power supplies were controlled using a computer program (LabView ${ }^{\circledR}$, National Instruments). Therewith the voltage was applied in a way that the line of zero potential laid along the crack. This was necessary for two reasons: the limited measuring range of the KFM-mode of $+/-10 \mathrm{~V}$, and due to the fact, that the topography of the surface is only recorded well, when the contact potential difference between the tip and the sample is close to zero. The measuring setup is shown in Fig. 2. The KFM-experiments were performed with a commer- 
cial SPM (Dimension3000, Nanoscope IV Controller, Veeco) using conducting, PtIr-coated, doped silicon tips with a resonant frequency around $75 \mathrm{~Hz}$ (PointProbe plus-tip for Electrostatic Force Microscopy PPP-EFM, Nanosensors). For the interleave scan, the lift height was set to $10-20 \mathrm{~nm}$. $6 \mathrm{~V}$ were taken for the driving voltage. The scan rate varied from $0.2 \mathrm{~Hz}$ for large scans $(60 \mu \mathrm{m})$ and $2 \mathrm{~Hz}$ for small scans $(5 \mu \mathrm{m})$, i.e. the scan speed was slightly higher for the larger scans.

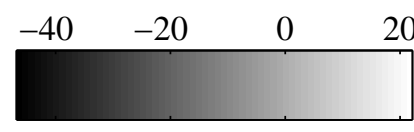

height $\mathrm{h} / \mathrm{nm}$
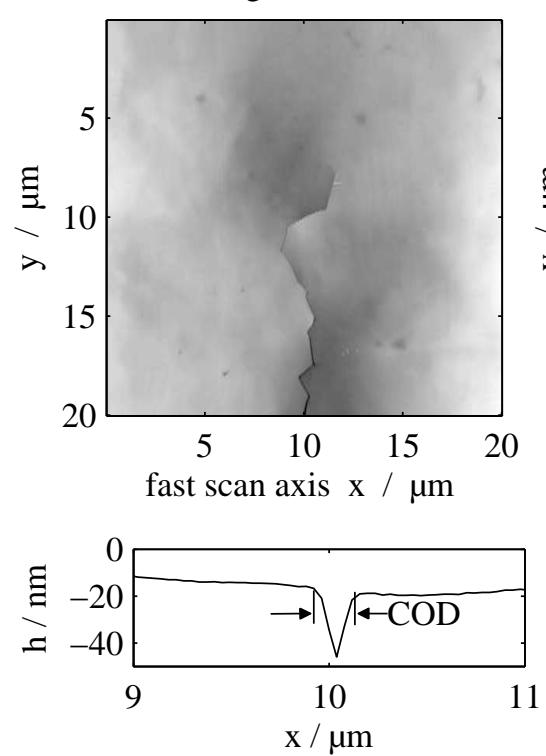

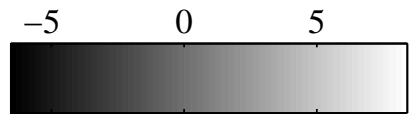

potential $\Phi / \mathrm{V}$
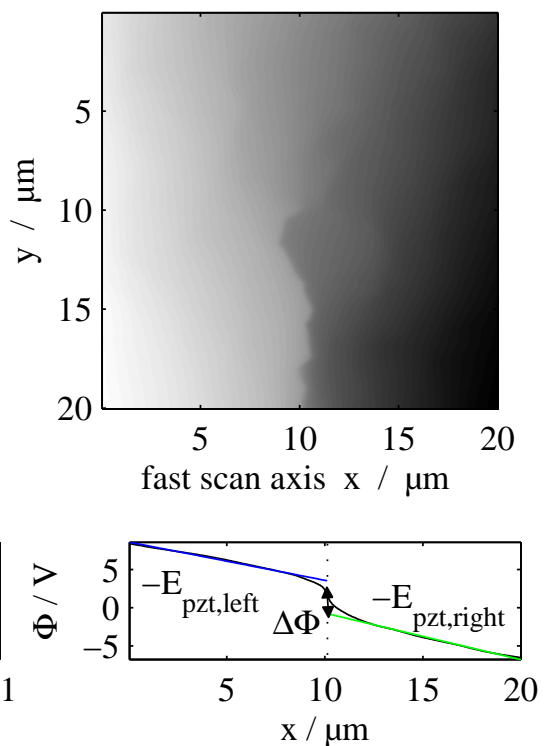

Fig. 3 Images of the topography (top-left) and the surface potential (top-right) of the scanned surface with an electric field of $400 \mathrm{~V} / \mathrm{mm}$ applied perpendicular to the crack (electric field and polarization vector from right to left). The lower graphs show the determination of the COD (bottom-left) and the electric field in the PZT and the potential drop across the crack.

Two data sets are received from every KFM measurement: the height (h) and the corresponding surface potential profile $(\Phi)$. Topographical images were processed using the microscope's software (NanoScope ${ }^{\circledR} 7.2$, Veeco) with a flattening function, which means that a polynomial is fitted to each data line and then subtracted to remove tilt and bow. KFM images were not post-processed. The images shown in Fig. 3 are exemplary and were taken with an applied field of $400 \mathrm{~V} / \mathrm{mm}$. The voltage steps in the KFM-images mark the location of the crack and reveal the lower dielectric constant of the crack compared to the ferroelectric material. The 
crack opening displacement COD, the electric field in the PZT $\mathrm{E}_{\mathrm{PZT}}$ and the potential drop across the crack $\Delta \Phi$ were determined as shown in the graphs in Fig. 3. The COD was determined using the microscope's image analysis software (NanoScope ${ }^{\circledR} 7.2$, Veeco) at positions where neither crack bridges nor kinks were present, i.e. where the crack faces were rather parallel to the crack direction. The distance from the crack tip $\mathrm{r}$ was determined graphically (CorelDRAW, Corel). To calculate the potential drop across the crack, the potential distribution was linearly extrapolated on both sides of the crack, and the distance of both lines was calculated at the inflection point of the potential curve (MATLAB ${ }^{2}$, Mathworks).

\section{Results}

Fig. 4 COD as a function of the distance from the crack tip $r$ at $\mathrm{E}_{\text {appl }}=0$ (main graph), and COD as a function of the applied electric field $\mathrm{E}_{\text {appl }}$ at $\mathrm{r}=5 \mu \mathrm{m}$ (inserted graph).

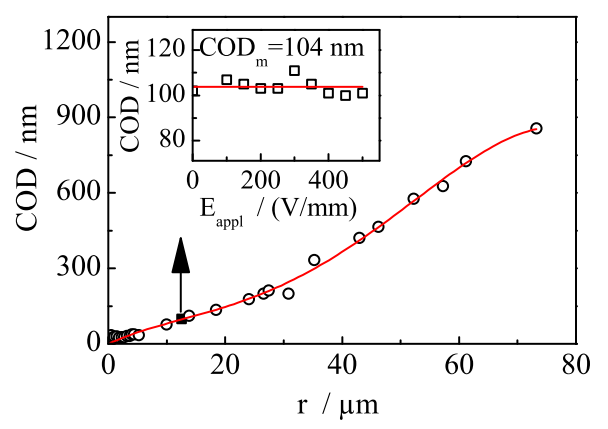

Since the measurement of the COD requires a high resolution, several overlapping scans were performed along the crack. The main graph of Fig. 4 shows the COD as a function of the distance from the crack tip $r$ at an applied electric field $E_{\text {appl }}=0 \mathrm{~V} / \mathrm{mm}$. In the inserted graph it is shown that, at a distance of $r=5 \mu \mathrm{m}$ from the tip, the COD was not influenced by $\mathrm{E}_{\mathrm{appl}}$.
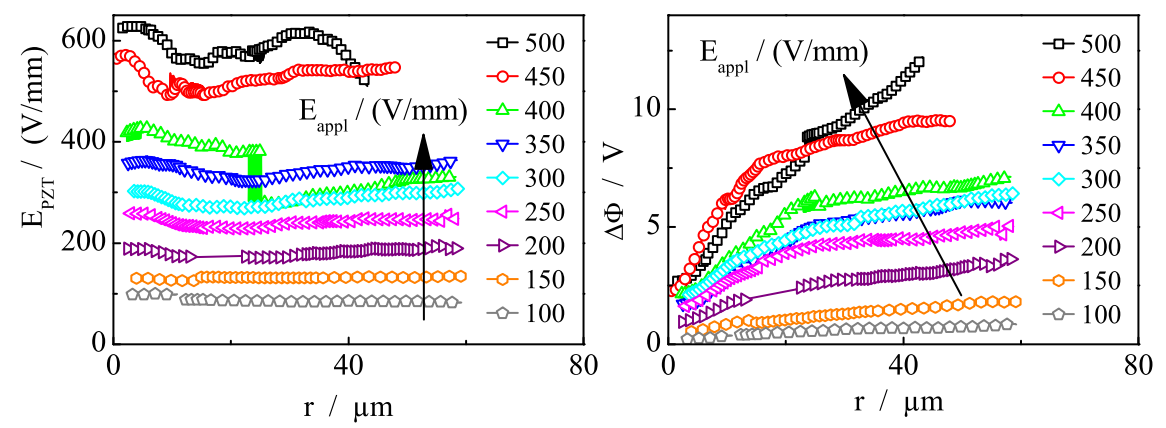

Fig. 5 Plots of the electric field in PZT (left) and the potential drop across the crack (right) as a function of the distance from the crack tip for different applied electric fields. 
The electric field in the PZT and the potential drop across the crack as a function of the distance from the crack tip are shown in Fig. 5 for the applied electric fields from 100 to $500 \mathrm{~V} / \mathrm{mm}$.

For the measurements with field reversal, the slow scan axis was disabled. Thus, the cantilever was kept at a constant distance from the crack tip during scanning. With the polarity of the applied voltage reversed, the potential distribution was measured as a function of time. The scanning frequency was chosen according to the scan size and the scan velocity. The scan size of $10 \mu \mathrm{m}$ was selected to be as small as possible, but large enough to allow a linear fitting of the potential on both sides of the crack to determine the electric field in the PZT. In KFM the potential is only measured every second scan in turn with the height. Fig. 6 shows the evolution of the potential plotted vs. time of an exemplary measurement, where the applied voltage was changed from $-100 \mathrm{~V} / \mathrm{mm}$ against poling direction to $100 \mathrm{~V} / \mathrm{mm}$ in poling direction, scanning with a frequency of $0.75 \mathrm{~Hz}$. The measurement was done at a distance of around $15 \mu \mathrm{m}$ from the rack tip; the COD at this distance was around $0.8 \mu \mathrm{m}$.

Fig. 6 3D plot showing the evolution of the surface potential with the time after the reversal of the applied field from $-100 \mathrm{~V} / \mathrm{mm}$ to $100 \mathrm{~V} / \mathrm{mm}$ during scanning at a constant distance from the crack tip ( $r=$ const.).
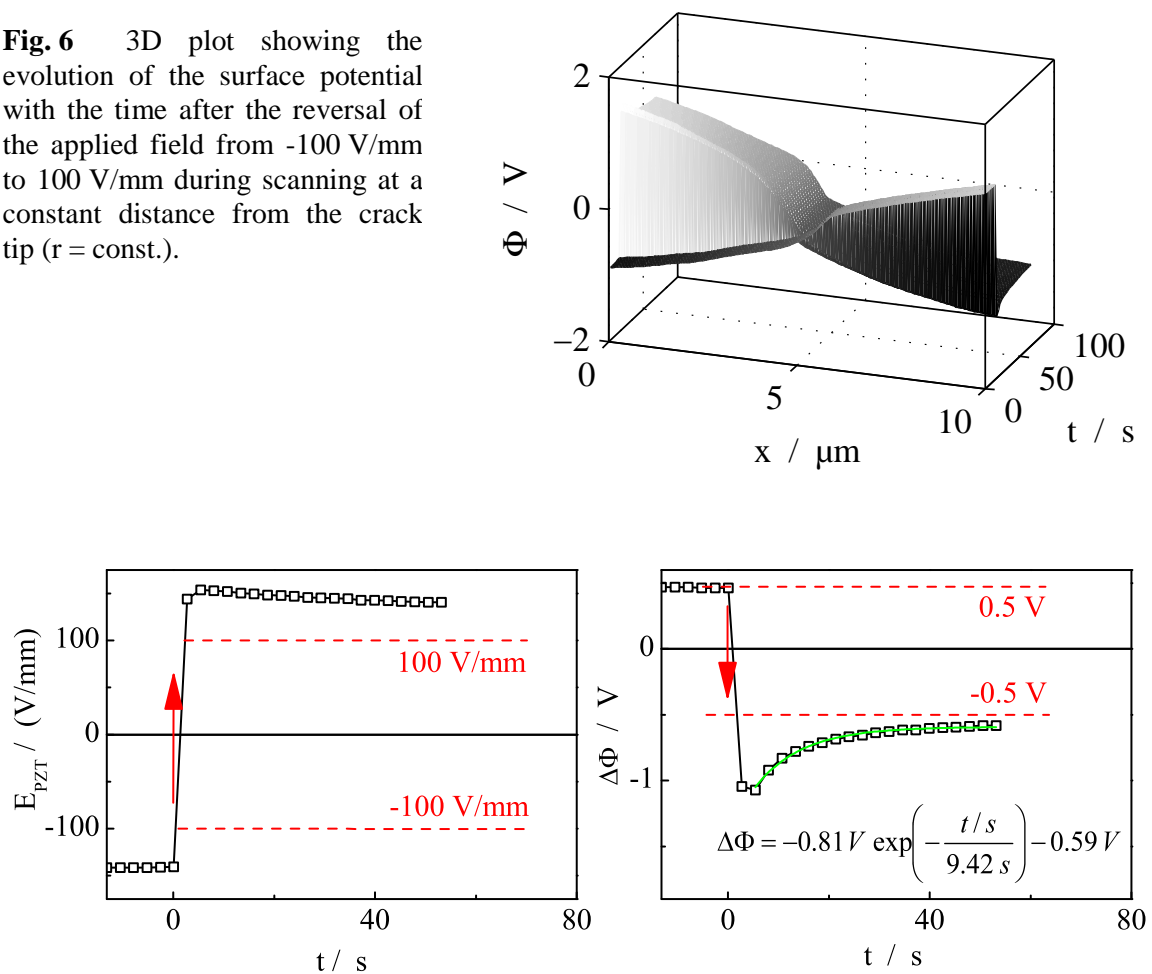

Fig. 7 Graph showing the electric field in the PZT (left) and the potential drop across the crack (right) as a function of time during the reversal of the applied voltage from -100 to $100 \mathrm{~V} / \mathrm{mm}$. 
Using the same procedure as above, the electric field in the PZT and the potential drop across the crack were calculated. As shown in Fig. 7 the electric field in the PZT changes rather step like, while the magnitude of the potential drop first increases before it reduces to the constant value with the opposite sign.

\section{Discussion}

For further analysis a very simple model was used: three capacitors in series connection representing a space filled with air between two layers of material with a dielectric constant of PZT. This model does not take into account the 3D shape of the Vickers crack system, the residual stresses around the indent, and the ferroelectric properties of PZT as well as the remnant polarization of the poled PZT. Using the condenser analogy the problem reduces to a $1 \mathrm{D}$ problem, which is fully described by the components of the electric field $\mathbf{E}$, dielectric displacement $\mathbf{D}$, and polarization $\mathbf{P}$ that are perpendicular to the crack plane. According to this model the electric field inside the crack $\mathrm{E}_{\text {crack }}$ was calculated by:

$$
\mathrm{E}_{\text {crack }}=\frac{\Delta \Phi}{\mathrm{COD}}
$$
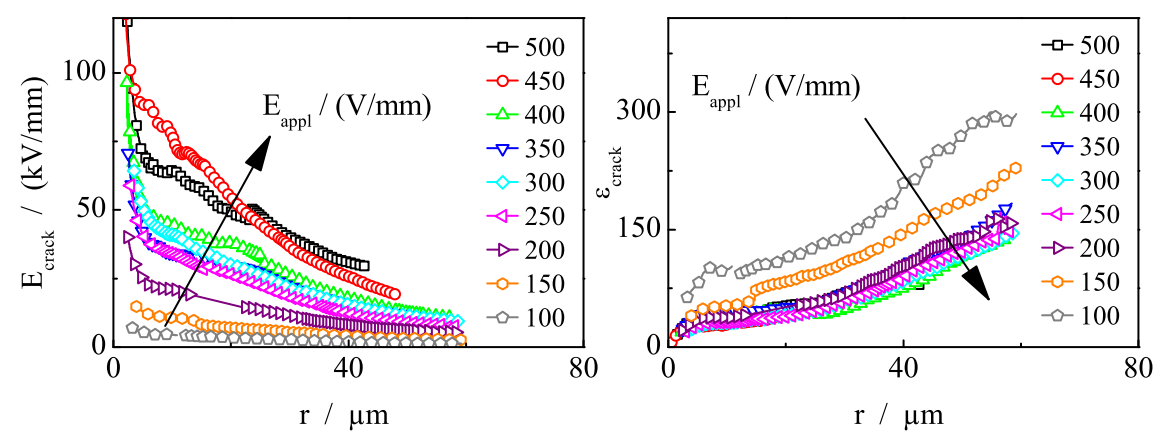

Fig. 8 Plots of the electric field across the crack (left) and the effective dielectric constant of the crack (right) as a function of the distance from the crack tip for different applied electric fields.

The results for the electric field in the crack as a function of the distance from the crack tip and the applied electric field are shown in the left graph of Fig. 8. The electric fields inside the crack are four orders of magnitude higher than in the PZT. Knowing the dielectric constant of the PZT $\varepsilon_{\mathrm{PZT}}$, either an effective dielectric constant of the crack $\varepsilon_{\text {crack, }}$ or an additional surface charge density on the crack surfaces $\omega_{\mathrm{s}}$ can be calculated using constitutive equations for the dielectric dis- 
placement D. Since the dielectric displacement is continuous across an interface between two different dielectric materials, at the crack surface it is:

$$
\mathrm{D}_{\mathrm{PZT}}=\varepsilon_{0} \varepsilon_{\mathrm{PZT}} \mathrm{E}_{\mathrm{PZT}}=\varepsilon_{0} \varepsilon_{\text {crack }} \mathrm{E}_{\text {crack }}=\varepsilon_{0} \mathrm{E}_{\text {crack }}+\omega_{\mathrm{s}} .
$$

The right graph in Fig. 8 shows that the effective dielectric constant rises with the distance from the crack tip and decreases with the applied electric field. For applied fields higher than $200 \mathrm{~V} / \mathrm{mm}$ a saturation value is reached. The values for $\varepsilon_{\text {crack }}$ range from around 10 near the tip to over 200 at a distance of $60 \mu \mathrm{m}$.

For every applied electric field $\varepsilon_{0} \mathrm{E}_{\text {crack }}$ is small compared to $\varepsilon_{0} \varepsilon_{\mathrm{PZT}} \mathrm{E}_{\mathrm{PZT}}$. Hence according to equation 2 , the free surface charges correspond to the dielectric displacement in the PZT for every applied field strength. Given that a very simple model was chosen to characterize the dielectric constant of the crack, the results of the measurements with a constant applied electric field show that neither a single dielectric constant nor a constant surface charge density on the crack surface can be used to describe the dielectric behavior of the whole crack.

For the time dependent measurement, the decay of the electric potential drop after the field reversal was fitted with an exponential function giving a time constant $\tau_{\Delta \Phi}$ of $9.5 \mathrm{~s}$ (Fig. 7, right):

$$
\Delta \Phi=-0.81 \mathrm{~V} \exp \left(-\frac{\mathrm{t} / \mathrm{s}}{9.42 \mathrm{~s}}\right)-0.59 \mathrm{~V}
$$

Furthermore the electric field inside the crack, the dielectric displacement in the PZT, the effective dielectric constant of the crack and the surface charge density were calculated. It was assumed that the surface charge density is a function of time. According to this assumption the dielectric displacement after the reversal of the voltage is described by:

$$
\mathrm{D}_{\mathrm{PZT}}(\mathrm{t})=\varepsilon_{0} \mathrm{E}_{\text {crack }}+\omega_{\mathrm{S}}(\mathrm{t})
$$

If the charge density on the crack surface and the dielectric displacement in the PZT are plotted vs. time (Fig. 9, right), it turns out that immediately after the reversal $\varepsilon_{0} \mathrm{E}_{\text {crack }} \ll<\mathrm{D}_{\text {PZT }}$. If $\varepsilon_{0} \mathrm{E}_{\text {crack }}$ can be neglected, $\omega_{\mathrm{s}} \approx \mathrm{D}_{\text {PZT }}$ according to eq. (4).

It was expected that directly after the field reversal, the surface charge redistribution $\omega_{\mathrm{s} \text {,model }}(\mathrm{t})$ could be described by an exponential equation,

$$
\varpi_{\mathrm{s}, \text { mod el }}(\mathrm{t})=2{\varpi_{\mathrm{s}}}^{0} \exp \left(-\frac{\mathrm{t}}{\tau}\right)-{\varpi_{\mathrm{s}}}^{0},
$$

where $\omega_{\mathrm{s}}{ }^{0}$ is the surface charge density before the field reversal. The expected progression of $\omega_{\mathrm{s} \text {,model }}$ is added in the plot of $\omega_{\mathrm{s}} \mathrm{vs}$. time. It is obvious that if there 
is an exponential time dependence of $\omega_{\mathrm{s}}$, the time constant $\tau_{\omega \mathrm{s}}$ is much smaller than $1 \mathrm{~s}$. The right graph in Fig. 9 shows first a drop in the effective dielectric constant of the crack at the moment of the reversal of the applied voltage and then a slow increase.
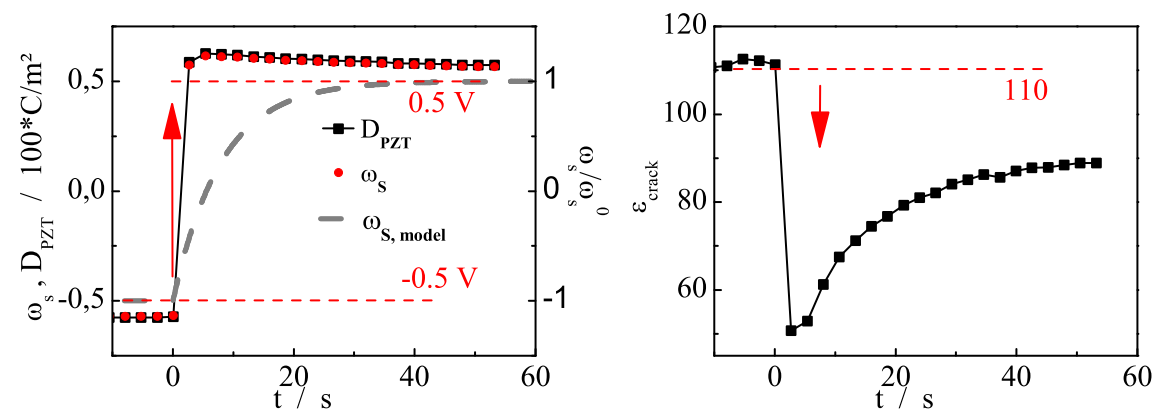

Fig. 9 Graph showing both the dielectric displacement in the PZT and the charge density on the crack surface (left) and the dielectric constant of the crack (right) as a function of time during the reversal of the applied voltage from -100 to $100 \mathrm{~V} / \mathrm{mm}$.

In summary this means: the experimental results suggest, that at least two time constants are necessary to describe the time dependent surface charge density on the crack surface. One, much smaller than one second, which can not be measured within the time resolution of the KFM method, and one in the range of $10 \mathrm{~s}$. The first one leads to an instantaneous screening of the bigger part of dielectric displacement in the PZT. The second one leads to slow exponential change of the potential drop.

To explain the charge transport to the crack surface, different approaches are discussed in the following. In time resolved measurements of the conductivity of PZT at 333 and $666 \mathrm{~V} / \mathrm{mm}$, a starting value of approximately $10^{-12} \mathrm{C} / \mathrm{Vms}$ was measured after $400 \mathrm{~ms}$. Assuming an electric conductivity $\sigma=10^{-12} \mathrm{C} / \mathrm{Vms}$ of PZT at an applied electric field of $100 \mathrm{~V} / \mathrm{mm}$ yields an electric current density of $\mathrm{j}=10^{-7} \mathrm{C} / \mathrm{m}^{2} \mathrm{~s}$. To compensate a dielectric displacement of $0.004 \mathrm{C} / \mathrm{m}^{2}$ it would take $11 \mathrm{~h}$ to carry the charge from the electrode to the crack surface. Therefore the change in the surface charge density can never be due to electrical conductivity in bulk PZT. Within one second also ferroelectric domain switching could lead to a redistribution of charges on the crack surface. Another approach to explain the behavior of the crack is the assumption of crack bridges. Crack bridges are discontinuous elements along a crack path, like contacting crack faces (frictional bridge) or a discontinuous crack path (elastic bridges). In a linear model an effective dielectric constant of the crack of 40 could be caused by a fraction of $\mathrm{f}=0.9 \%$ crack bridges per unit area, which to contacting crack faces: 


$$
\varepsilon_{\text {crack }}=\mathrm{f} \varepsilon_{\mathrm{PZT}}+(1-\mathrm{f}), \mathrm{f}=\frac{\varepsilon_{\text {crack }}-1}{\varepsilon_{\mathrm{PZT}}-1}=\frac{39}{4499}=0,0087=0,9 \% \text {. }
$$

The images of the samples taken with the optical microscope and the scanning probe microscope prove the existence of elastic and frictional crack bridges. The exemplary images in Fig. 1 show a typical crack path. Although the grain boundaries cannot be seen, the crack path suggests intergranular fracture (tortuous path around grains). The crack profile also shows micro cracking and crack bridging (elastic and frictional bridges). Fig. 10 shows an exemplary SPM image of a small part of a crack path with a frictional bridge. An argument against the explanation of $\varepsilon_{\text {crack }}$ by crack bridges is the fact that $\varepsilon_{\text {crack }}$ rises with larger distance from the crack tip, although the crack bridges should get less due to the increasing crack opening displacement.

Fig. 10 SPM image of the topography (amplitude signal of the height) showing a frictional bridge.

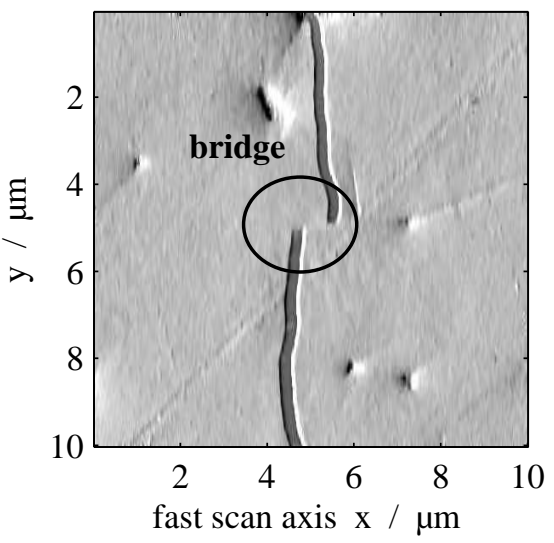

\section{Conclusions}

Electric field dependent measurements of the crack opening profile and the electrical potential around a Vickers indentation crack in ferroelectric PZT confirm the KFM measurements from Schneider et al. [12] that the potential drop across the crack is much smaller than expected for a crack filled with air with a dielectric constant of 1 . If no surface charges are assumed, the calculated the calculated effective dielectric constant based on a simple model increases from around 10 near the crack tip to 200 at a distance of $60 \mu \mathrm{m}$ from the crack tip, and decreases slightly and continuously with the applied electric field. If a dielectric constant of 1 inside the crack is assumed, the calculated crack surface charge densities are almost identical with the dielectric displacement in the PZT ceramic. 
Time resolved measurements showed that the bigger part of the calculated charge density on the crack surfaces follows the electric field instantaneously. An estimate shows that the electrical conductivity of the PZT is too low to change the surface charges within a few seconds by electrical conduction through the bulk PZT. On the other hand, it could be shown that experimentally observed grain bridges could explain the effective dielectric constant of much bigger than 1 and the fast charge displacement after the reversal of the applied electric field, although they cannot explain the increasing effective dielectric constant of the crack with increasing distance from the crack tip.

Acknowledgements: We thank Rodrigo Pacher Fernandez and Claudia Neusel for the measurement of the electrical conductivity of PZT and the DFG (German Science Foundation) for supporting this project under the grant number SCHN 372/12-2.

\section{References}

[1] Balke H., Kemmer G. \& Drescher J., Some remarks on the fracture mechanics of piezoelectric solids. MicroMaterials Conference "MicroMat 1997", 1997, 398 - 401.

[2] Hao T.H. \& Shen Z.Y., A New Electric Boundary-Condition of Electric FractureMechanics and Its Applications. Eng. Fract. Mech., 1994, 47(6), 793-802.

[3] McMeeking R.M., The energy release rate for a Griffith crack in a piezoelectric material. Eng. Fract. Mech., 2004, 71(7-8), 1149-1163.

[4] Landis C.M., Energetically consistent boundary conditions for electromechanical fracture. International Journal of Solids and Structures, 2004, 41(22-23), 6291-6315.

[5] Landis C.M., Energetically consistent boundary conditions for electromechanical fracture (Erratum, vol 41, pg 6291, 2004). International Journal of Solids and Structures, 2005, 42(8), 2461-2463.

[6] Li W.Y., McMeeking R.M. \& Landis C.M., On the crack face boundary conditions in electromechanical fracture and an experimental protocol for determining energy release rates. Eur. J. Mech. A-Solids, 2008, 27(3), 285-301.

[7] Jelitto H., Felten F., Swain M.V., Balke H. \& Schneider G.A., Measurement of the total energy release rate for cracks in PZT under combined mechanical and electrical loading. J. Appl. Mech.-Trans. ASME, 2007, 74(6), 1197-1211.

[8] Jelitto H., Felten F., Hausler C., Kessler H., Balke H. \& Schneider G.A., Measurement of energy release rates for cracks in PZT under electromechanical loads. J. European Ceram. Soc., 2005, 25(12), 2817-2820.

[9] Jelitto H., Kessler H., Schneider G.A. \& Balke H., Fracture behavior of poled piezoelectric PZT under mechanical and electrical loads. J. European Ceram. Soc., 2005, 25(5), 749-757.

[10] Häusler C., Jelitto H., Neumeister P., Balke H. \& Schneider G.A., Interfacial fracture of piezoelectric multilayer actuators under mechanical and electrical loading. Int. J. Fract., 2009, 160(1), 43-54.

[11] Haug A. \& McMeeking R.M., Cracks with surface charge in poled ferroelectrics. Eur. J. Mech. A-Solids, 2006, 25(1), 24-41.

[12] Schneider G.A., Felten F. \& McMeeking R.M., The electrical potential difference across cracks in PZT measured by Kelvin Probe Microscopy and the implications for fracture. Acta Mater., 2003, 51(8), 2235-2241. 
[13] Felten F. PhD Thesis: Anwendung der Rastersondenmikroskopie zur Bestimmung bruchmechanischer Parameter und lokaler piezoelektrischer Eigenschaften von Ferroelektrika. book series: Berichte aus der Materialwissenschaft, Shaker Verlag, Aachen, 2006.

[14] Kalinin S.V. \& Bonnell D.A., Local potential and polarization screening on ferroelectric surfaces. Phys. Rev. B, 2001, 63(12),

[15] Jacobs H.O., Leuchtmann P., Homan O.J. \& Stemmer A., Resolution and contrast in Kelvin probe force microscopy. Journal of Applied Physics, 1998, 84(3), 1168-1173.

[16] JohnsonMatthey, Piezoceramic Masses. 2009, online available from: http://ect.jmcatalysts.com/ or http://www.piezoproducts.com/de/.

[17] Lawn B.R. Fracture of brittle solids. book series: Cambridge solid state science series, 2 ed., Cambridge University Press, Cambridge, 1993. 